\title{
Weighted Weak Local Hardy Spaces Associated with Schrödinger Operators
}

\author{
Hua Zhu \\ Beijing International Studies University, Beijing 100024, China \\ Correspondence should be addressed to Hua Zhu; zhuhua@pku.edu.cn
}

Received 11 April 2015; Revised 30 June 2015; Accepted 13 July 2015

Academic Editor: Guozhen Lu

Copyright (C) 2015 Hua Zhu. This is an open access article distributed under the Creative Commons Attribution License, which permits unrestricted use, distribution, and reproduction in any medium, provided the original work is properly cited.

We characterize the weighted weak local Hardy spaces $\mathrm{Wh}_{\rho}^{p}(\omega)$ related to the critical radius function $\rho$ and weights $\omega \in A_{\infty}^{\rho, \infty}\left(\mathbb{R}^{n}\right)$ which locally behave as Muckenhoupt's weights and actually include them, by the atomic decomposition. As an application, we show that localized Riesz transforms are bounded on the weighted weak local Hardy spaces.

\section{Introduction}

The theory of classical local Hardy spaces, originally introduced by Goldberg [1], plays an important role in various fields of analysis and partial differential equations; see [2-7] and their references. Huy Qui [2] studied the weighted version $h_{\omega}^{p}$ of the local Hardy spaces $h^{p}$ considered by Goldberg, where the weighted $\omega$ belongs to the Muckenhoupt class. In [8], Rychkov introduced and studied some properties of the weighted Besov-Lipschitz spaces and Triebel-Lizorkin spaces with weights that are locally in $A_{p}\left(\mathbb{R}^{n}\right)$ (Muckenhoupt's weights, see [4, 9-11]) but may grow or decrease exponentially. In [12], Tang established the weighted atomic decomposition characterization of the weighted local Hardy space $h_{\omega}^{p}\left(\mathbb{R}^{n}\right)$ with local weights. Recently, in [13], the authors established weighted atomic decomposition characterizations for weighted local Hardy spaces $h_{\rho}^{p}(\omega)$ with $\omega \in A_{p}^{\rho, \theta}\left(\mathbb{R}^{n}\right)$.

On the other hand, the weak $H^{1}$ space theory was first introduced by Fefferman and Soria in [14]. Then the weak $H^{p}$ $(0<p<1)$ space theory was studied by Liu in [15]. Recently, Tang [16] established the weighted weak local Hardy space $\mathrm{Wh}_{\omega}^{p}\left(\mathbb{R}^{n}\right)$ with local weights.

The purpose of this paper is twofold. The first goal is to characterize weighted weak local Hardy spaces by atomic decomposition. The second goal is to show that localized Riesz transforms are bounded on weighted weak local Hardy spaces.
The paper is organized as follows. In Section 2, we introduce some notation and properties concerning weights and grand maximal functions. In Section 3, we establish weighted atomic decomposition of weighted weak local Hardy spaces with $\omega \in A_{p}^{\rho, \theta}\left(\mathbb{R}^{n}\right)$. Finally, in Section 4, we show that localized Riesz transforms are bounded on weighted weak local Hardy spaces.

Throughout this paper, we let $C$ denote constants that are independent of the main parameters involved but whose value may differ from line to line. By $A \sim B$, we mean that there exists a constant $C>1$ such that $1 / C \leq A / B \leq C$. The symbol $A \lesssim B$ means that $A \leq C B$. The symbol $[s]$ for $s \in \mathbb{R}$ denotes the maximal integer no more than $s$. We also set $\mathbb{N} \equiv\{1,2, \ldots\}$ and $\mathbb{Z}_{+} \equiv \mathbb{N} \cup\{0\}$. The multiindex notation is usual: for $\alpha=\left(\alpha_{1}, \ldots, \alpha_{n}\right)$ and $\partial^{\alpha}=\left(\partial / \partial_{x_{1}}\right)^{\alpha_{1}} \ldots\left(\partial / \partial_{x_{n}}\right)^{\alpha_{n}}$.

\section{Preliminaries}

In this section, we review some notions and notations concerning the weight classes $A_{p}^{\rho, \theta}\left(\mathbb{R}^{n}\right)$ introduced in [17-19]. Given $B=B(x, r)$ and $\lambda>0$, we will write $\lambda B$ for the $\lambda$-dilate ball, which is the ball with the same center $x$ and with radius $\lambda r$. Similarly, $Q(x, r)$ denotes the cube centered at $x$ with side length $r$ (here and below only cubes with sides parallel to the axes are considered), and $\lambda Q(x, r)=Q(x, \lambda r)$. Particulalry, we will denote $2 B$ by $B^{*}$ and $2 Q$ by $Q^{*}$. 
Let $\mathscr{L}=-\Delta+V$ be a Schrödinger operator on $\mathbb{R}^{n}, n \geq 3$, where $V \not \equiv 0$ is a fixed nonnegative potential. We assume that $V$ belongs to the reverse Hölder class $\mathrm{RH}_{s}\left(\mathbb{R}^{n}\right)$ for some $s \geq n / 2$; that is, there exists $C=C(s, V)>0$ such that

$$
\left(\frac{1}{|B|} \int_{B} V(x)^{s} d x\right)^{1 / s} \leq C\left(\frac{1}{|B|} \int_{B} V(x) d x\right),
$$

for every ball $B \subset \mathbb{R}^{n}$. Trivially, $\mathrm{RH}_{q}\left(\mathbb{R}^{n}\right) \subset \mathrm{RH}_{p}\left(\mathbb{R}^{n}\right)$ provided that $1<p \leq q<\infty$. It is well known that if $V \in \mathrm{RH}_{q}\left(\mathbb{R}^{n}\right)$ for some $q>1$, then there exists $\varepsilon>0$, which depends only on $d$ and the constant $C$ in above inequality such that $V \in \mathrm{RH}_{q+\varepsilon}\left(\mathbb{R}^{n}\right)$ (see [20]). Moreover, the measure $V(x) d x$ satisfies the doubling condition:

$$
\int_{B(y, 2 r)} V(x) d x \leq C \int_{B(y, r)} V(x) d x .
$$

With regard to the Schrödinger operator $\mathscr{L}$, we know that the operators derived from $\mathscr{L}$ behave "locally" quite similar to those corresponding to the Laplacian (see [21, 22]). The notion of locality is given by the critical radius function. Consider

$$
\rho(x)=\frac{1}{m_{V}(x)}=\sup _{r>0}\left\{r: \frac{1}{r^{n-2}} \int_{B(x, r)} V(y) d y \leq 1\right\} .
$$

Throughout the paper we assume that $V \not \equiv 0$, so that $0<$ $\rho(x)<\infty$ (see [22]). In particular, $m_{V}(x)=1$ with $V=1$ and $m_{V}(x) \sim(1+|x|)$ with $V=|x|^{2}$.

Lemma 1 (see [22]). There exist $C_{0} \geq 1$ and $k_{0} \geq 1$ so that for all $x, y \in \mathbb{R}^{n}$

$$
\begin{gathered}
C_{0}^{-1} \rho(x)\left(1+\frac{|x-y|}{\rho(x)}\right)^{-k_{0}} \leq \rho(y) \\
\leq C_{0} \rho(x)\left(1+\frac{|x-y|}{\rho(x)}\right)^{k_{0} /\left(k_{0}+1\right)}
\end{gathered}
$$

In particular, $\rho(x) \sim \rho(y)$ when $y \in B(x, r)$ and $r \leq C \rho(x)$, where $C$ is a positive constant.

A ball of the form $B(x, \rho(x))$ is called critical, and in what follows we will call any positive continuous function $\rho$ that satisfies (3) critical radius function, not necessarily coming from a potential $V$. Clearly, if $\rho$ is such a function, so it is $\beta \rho$ for any $\beta>0$. As a consequence of the above lemma we acquire the following result.

Lemma 2 (see [23]). There exists a sequence of points $x_{j} \in \mathbb{R}^{n}$, $j \geq 1$, such that the family $B_{j}=B\left(x_{j}, \rho\left(x_{j}\right)\right), j \geq 1$, satisfies the fact that

(1) $\bigcup_{j} B_{j}=\mathbb{R}^{n}$;

(2) for every $\sigma \geq 1$ there exist constants $C$ and $N_{1}$ such that $\Sigma_{j} \chi_{\sigma B_{j}} \leq C \sigma^{N_{1}}$.

In this paper, we write $\Psi_{\theta}(B)=\left(1+r / \rho\left(x_{0}\right)\right)^{\theta}$, where $\theta \geq$ $0 ; x_{0}$ and $r$ denote the center and radius of $B$, respectively.
A weight always refers to a positive function which is locally integrable. As in [17], we say that a weight $\omega$ belongs to the class $A_{p}^{\rho, \theta}\left(\mathbb{R}^{n}\right)$ for $1<p<\infty$ if there is a constant $C$ such that for all balls $B$. One has

$$
\begin{aligned}
& \left(\frac{1}{\Psi_{\theta}(B)|B|} \int_{B} \omega(y) d y\right) \\
& \quad \cdot\left(\frac{1}{\Psi_{\theta}(B)|B|} \int_{B} \omega^{-1 /(p-1)}(y) d y\right)^{p-1} \leq C .
\end{aligned}
$$

We also say that a nonnegative function $\omega$ satisfies the $A_{1}^{\rho, \theta}\left(\mathbb{R}^{n}\right)$ condition if there exists a constant $C$ such that

$$
M_{V, \theta}(\omega)(x) \leq C \omega(x), \quad \text { a.e. } x \in \mathbb{R}^{n}
$$

where

$$
M_{V, \theta} f(x) \equiv \sup _{x \in B} \frac{1}{\Psi_{\theta}(B)|B|} \int_{B}|f(y)| d y .
$$

When $V=0$, we denote $M_{0} f(x)$ by $M f(x)$ (the standard Hardy-Littlewood maximal function). It is easy to see that $|f(x)| \leq M_{V, \theta} f(x) \leq M f(x)$ for a.e. $x \in \mathbb{R}^{n}$ and any $\theta \geq 0$.

Clearly, the classes $A_{p}^{\rho, \theta}$ are increasing with $\theta$, and we denote $A_{p}^{\rho, \infty}=\bigcup_{\theta \geq 0} A_{p}^{\rho, \theta}$. By Hölder's inequality, we see that $A_{p_{1}}^{\rho, \theta} \subset A_{p_{2}}^{\rho, \theta}$, if $1 \leq p_{1}<p_{2}<\infty$, and we also denote $A_{\infty}^{\rho, \infty}=$ $\bigcup_{p \geq 1} A_{p}^{\rho, \infty}$. In addition, for $1 \leq p \leq \infty$, denote by $p^{\prime}$ the adjoint number of $p$; that is, $1 / p+1 / p^{\prime}=1$.

Since $\Psi_{\theta}(B) \geq 1$ with $\theta \geq 0$, then $A_{p} \subset A_{p}^{\rho, \theta}$ for $1 \leq p<$ $\infty$, where $A_{p}$ denotes the classical Muckenhoupt weights; see $[10,24]$. Moreover, the inclusions are proper. In fact, as the example given in [18], let $\theta>0$ and $0 \leq \gamma \leq \theta$; it is easy to check that $\omega(x)=(1+|x|)^{-(n+\gamma)} \notin A_{\infty}=\bigcup_{p \geq 1} A_{p}$ and $\omega(x) d x$ is not a doubling measure, but $\omega(x)=(1+|x|)^{-(n+\gamma)} \epsilon$ $A_{1}^{\rho, \theta}$ provided that $V=1$ and $\Psi_{\theta}\left(B\left(x_{0}, r\right)\right)=(1+r)^{\theta}$.

In what follows, given a Lebesgue measurable set $E$ and a weight $\omega,|E|$ will denote the Lebesgue measure of $E$ and $\omega(E):=\int_{E} \omega(x) d x$. For any $\omega \in A_{\infty}^{\rho, \infty}$, the space $L_{\omega}^{p}\left(\mathbb{R}^{n}\right)$ with $p \in(0, \infty)$ denotes the set of all measurable functions $f$ such that

$$
\|f\|_{L_{\omega}^{p}\left(\mathbb{R}^{n}\right)} \equiv\left(\int_{\mathbb{R}^{n}}|f(x)|^{p} \omega(x) d x\right)^{1 / p}<\infty,
$$

and $L_{\omega}^{\infty}\left(\mathbb{R}^{n}\right) \equiv L^{\infty}\left(\mathbb{R}^{n}\right)$. The symbol $L_{\omega}^{p, \infty}\left(\mathbb{R}^{n}\right)$ denotes the set of all measurable functions $f$ such that

$$
\begin{aligned}
\|f\|_{L_{\omega}^{p, \infty}\left(\mathbb{R}^{n}\right)} & \equiv\left(\sup _{\lambda>0}\left\{\lambda^{p} \omega\left(\left\{x \in \mathbb{R}^{n}:|f(x)|>\lambda\right\}\right)\right\}\right)^{1 / p} \\
& <\infty .
\end{aligned}
$$

We define the local Hardy-Littlewood maximal operator by

$$
M^{\text {loc }} f(x) \equiv \sup _{\substack{x \in B\left(x_{0}, r\right) \\ r \leq \rho\left(x_{0}\right)}} \frac{1}{|B|} \int_{B}|f(y)| d y .
$$


We remark that balls can be replaced by cubes in definition of $A_{p}^{\rho, \theta}$ and $M_{V, \theta}$, since $\Psi(B) \leq \Psi(2 B) \leq 2^{\theta} \Psi(B)$. In fact, for the cube $Q=Q\left(x_{0}, r\right)$, we can also define $\Psi_{\theta}(Q)=$ $\left(1+r / \rho\left(x_{0}\right)\right)^{\theta}$.

Next, we give some properties of weights class $A_{p}^{\rho, \theta}$ for $p \geq 1$.

Lemma 3. Let $\omega \in A_{p}^{\rho, \infty}=\bigcup_{\theta \geq 0} A_{p}^{\rho, \theta}$ for $p \geq 1$. Then

(i) if $1 \leq p_{1}<p_{2}<\infty$, then $A_{p_{1}}^{\rho, \theta} \subset A_{p_{2}}^{\rho, \theta}$;

(ii) $\omega \in A_{p}^{\rho, \theta}$ if and only if $\omega^{-1 /(p-1)} \in A_{p^{\prime}}^{\rho, \theta}$, where $1 / p+$ $1 / p^{\prime}=1 ;$

(iii) if $\omega \in A_{p}^{\rho, \infty} ; 1<p<\infty$, then there exists $\epsilon>0$ such that $\omega \in A_{p-\epsilon}^{\rho, \infty}$;

(iv) let $f \in L_{\text {loc }}\left(\mathbb{R}^{n}\right), 0<\delta<1$, then $\left(M_{V, \theta} f\right)^{\delta} \in A_{1}^{\rho, \theta}$;

(v) let $1<p<\infty$, then $\omega \in A_{p}^{\rho, \infty}$ if and only if $\omega=$ $\omega_{1} \omega_{2}^{1-p}$, where $\omega_{1}, \omega_{2} \in A_{1}^{\rho, \infty}$;

(vi) for $\omega \in A_{p}^{\rho, \theta}, Q=Q(x, r)$ and $\lambda>1$, there exists a positive constant $C$ such that

$$
\omega(\lambda Q) \leq C\left(\Psi_{\theta}(\lambda Q)\right)^{p} \lambda^{n p} \omega(Q) ;
$$

(vii) if $p \in(1, \infty)$ and $\omega \in A_{p}^{\rho, \theta}\left(\mathbb{R}^{n}\right)$, then the local HardyLittlewood maximal operator $M^{\text {loc }}$ is bounded on $L_{\omega}^{p}\left(\mathbb{R}^{n}\right)$;

(viii) if $\omega \in A_{1}^{\rho, \theta}\left(\mathbb{R}^{n}\right)$, then $M^{\text {loc }}$ is bounded from $L_{\omega}^{1}\left(\mathbb{R}^{n}\right)$ to $L_{\omega}^{1, \infty}\left(\mathbb{R}^{n}\right)$.

Proof. (i)-(viii) have been proved in [17, 19].

For any $\omega \in A_{\infty}^{\rho, \infty}\left(\mathbb{R}^{n}\right)$, define the critical index of $\omega$ by

$$
q_{\omega} \equiv \inf \left\{p \in[1, \infty): \omega \in A_{p}^{\rho, \infty}\left(\mathbb{R}^{n}\right)\right\} .
$$

Obviously, $q_{\omega} \in[1, \infty)$. If $q_{\omega} \in(1, \infty)$, then $\omega \notin A_{q_{\omega}}^{\rho, \infty}$, but $\omega \in A_{q_{\omega}+\varepsilon}^{\rho, \infty}$ for any $\varepsilon>0$.

The symbols $\mathscr{D}\left(\mathbb{R}^{n}\right)=C_{0}^{\infty}\left(\mathbb{R}^{n}\right), \mathscr{D}^{\prime}\left(\mathbb{R}^{n}\right)$ are the dual space of $\mathscr{D}\left(\mathbb{R}^{n}\right)$. For any $\varphi \in \mathscr{D}\left(\mathbb{R}^{n}\right)$, let $\varphi_{t}(x)=t^{-n} \varphi(x / t)$ for $t>0$ and $\varphi_{j}(x)=2^{j n} \varphi\left(2^{j} x\right)$ for $j \in \mathbb{Z}$.

Lemma 4 (see [13]). Let $\omega \in A_{\infty}^{\rho, \infty}\left(\mathbb{R}^{n}\right)$ and $q_{\omega}$ be as in (12) and $p \in\left(q_{\omega}, \infty\right]$.

(i) If $1 / p+1 / p^{\prime}=1$, then $\mathscr{D}\left(\mathbb{R}^{n}\right) \subset L_{\omega^{-1 /(p-1)}}^{p^{\prime}}\left(\mathbb{R}^{n}\right)$.

(ii) $L_{\omega}^{p}\left(\mathbb{R}^{n}\right) \subset \mathscr{D}^{\prime \prime}\left(\mathbb{R}^{n}\right)$ and the inclusion is continuous.
We now introduce some local maximal functions. For $N \in \mathbb{Z}_{+}$and $R \in(0, \infty)$, let

$$
\begin{gathered}
\mathscr{D}_{N, R}\left(\mathbb{R}^{n}\right) \equiv\left\{\varphi \in \mathscr{D}\left(\mathbb{R}^{n}\right): \operatorname{supp}(\varphi)\right. \\
\subset B(0, R),\|\varphi\|_{\mathscr{D}_{N}\left(\mathbb{R}^{n}\right)} \\
\left.\equiv \sup _{x \in \mathbb{R}^{n}} \sup _{\alpha \in \mathbb{Z}_{+}^{n},|\alpha| \leq N}\left|\partial^{\alpha} \varphi(x)\right| \leq 1\right\} .
\end{gathered}
$$

Definition 5. Let $N \in \mathbb{Z}_{+}$and $R \in(0, \infty)$. For any $f \in$ $\mathscr{D}^{\prime}\left(\mathbb{R}^{n}\right)$, the local nontangential grand maximal function $\widetilde{M}_{N, R}(f)$ of $f$ is defined by setting, for all $x \in \mathbb{R}^{n}$,

$$
\begin{aligned}
& \widetilde{\mathscr{M}}_{N, R}(f)(x) \equiv \sup \left\{\left|\varphi_{l} * f(z)\right|:|x-z|<2^{-l}\right. \\
& \left.\quad<\rho(x), \varphi \in \mathscr{D}_{N, R}\left(\mathbb{R}^{n}\right)\right\},
\end{aligned}
$$

and the local vertical grand maximal function $\mathscr{M}_{N, R}(f)$ of $f$ is defined by setting, for all $x \in \mathbb{R}^{n}$,

$$
\begin{aligned}
& \mathscr{M}_{N, R}(f)(x) \equiv \sup \left\{\left|\varphi_{l} * f(x)\right|: 0<2^{-l}<\rho(x), \varphi\right. \\
& \left.\quad \in \mathscr{D}_{N, R}\left(\mathbb{R}^{n}\right)\right\} .
\end{aligned}
$$

For convenience's sake, when $R=1$, we denote $\mathscr{D}_{N, R}\left(\mathbb{R}^{n}\right)$, $\widetilde{\mathscr{M}}_{N, R}(f)$, and $\mathscr{M}_{N, R}(f)$ simply by $\mathscr{D}_{N}^{0}\left(\mathbb{R}^{n}\right), \widetilde{\mathscr{M}}_{N}^{0}(f)$, and $\mathscr{M}_{N}^{0}(f)$, respectively; when $R=\max \left\{R_{1}, R_{2}, R_{3}\right\}>1$ (in which $R_{1}, R_{2}$, and $R_{3}$ are defined as in Lemmas 4.2, 4.4, and 4.8 in [13]), we denote $\mathscr{D}_{N, R}\left(\mathbb{R}^{n}\right), \widetilde{\mathscr{M}}_{N, R}(f)$, and $\mathscr{M}_{N, R}(f)$ simply by $\mathscr{D}_{N}\left(\mathbb{R}^{n}\right), \widetilde{\mathscr{M}}_{N}(f)$, and $\mathscr{M}_{N}(f)$, respectively. For any $N \in \mathbb{Z}_{+}$and $x \in \mathbb{R}^{n}$, obviously,

$$
\mathscr{M}_{N}^{0}(f)(x) \leq \mathscr{M}_{N}(f)(x) \leq \widetilde{\mathscr{M}}_{N}(f)(x) .
$$

Definition 6. Let

$$
\psi_{0} \in \mathscr{D}\left(\mathbb{R}^{n}\right) \quad \text { with } \int_{\mathbb{R}^{n}} \psi_{0}(x) d x \neq 0 .
$$

For every $x \in \mathbb{R}^{n}$, there exists an integer $j_{x} \in \mathbb{Z}$ satisfying $2^{-j_{x}}<\rho(x) \leq 2^{-j_{x}+1}$, and then for $j \geq j_{x}, A, B \in[0, \infty)$ and $y \in \mathbb{R}^{n}$, let $m_{j, A, B, x}(y) \equiv\left(1+2^{j}|y|\right)^{A} 2^{B|y| / \rho(x)}$.

The local vertical maximal function $\psi_{0}^{+}(f)$ of $f$ associated with $\psi_{0}$ is defined by setting, for all $x \in \mathbb{R}^{n}$,

$$
\psi_{0}^{+}(f)(x) \equiv \sup _{j \geq j_{x}}\left|\left(\psi_{0}\right)_{j} * f(x)\right|,
$$

the local tangential Peetre-type maximal function $\psi_{0, A, B}^{* *}(f)$ of $f$ associated with $\psi_{0}$ is defined by setting, for all $x \in \mathbb{R}^{n}$,

$$
\psi_{0, A, B}^{* *}(f)(x) \equiv \sup _{j \geq j_{x}, y \in \mathbb{R}^{n}} \frac{\left|\left(\psi_{0}\right)_{j} * f(x-y)\right|}{m_{j, A, B, x}(y)} .
$$

Obviously, for any $x \in \mathbb{R}^{n}$, we have $\psi_{0}^{+}(f)(x) \lesssim \psi_{0, A, B}^{* *}(f)(x)$. 
For $f \in L_{\mathrm{loc}}^{1}\left(\mathbb{R}^{n}\right), B \in[0, \infty)$, and $x \in \mathbb{R}^{n}$, let

$$
K_{B} f(x) \equiv \frac{1}{(\rho(x))^{n}} \int_{\mathbb{R}^{n}}|f(y)| 2^{-B(|x-y| / \rho(x))} d y,
$$

and for the operator $K_{B}$, we have the following lemma.

Lemma 7 (see [13]). Let $p \in(1, \infty)$ and $\omega \in A_{p}^{\rho, \theta}\left(\mathbb{R}^{n}\right)$; then there exist constants $C>0$ and $B_{0} \equiv B_{0}(\omega, n)>0$ such that, for all $B>B_{0} / p$,

$$
\left\|K_{B} f\right\|_{L_{\omega}^{p}\left(\mathbb{R}^{n}\right)} \leq C\|f\|_{L_{\omega}^{p}\left(\mathbb{R}^{n}\right)},
$$

for all $f \in L_{\omega}^{p}\left(\mathbb{R}^{n}\right)$.

\section{Proposition 8. Let $N \geq 2$. Then}

(i) there exists a positive constant $C$ such that, for all $f \in$ $L_{\text {loc }}^{1}\left(\mathbb{R}^{n}\right) \cap \mathscr{D}^{\prime}\left(\mathbb{R}^{n}\right)$ and almost every $x \in \mathbb{R}^{n}$,

$$
|f(x)| \leq \mathscr{M}_{N}^{0}(f)(x) \leq C M^{l o c}(f)(x) ;
$$

(ii) if $\omega \in A_{q}^{\rho, \infty}\left(\mathbb{R}^{n}\right)$ with $q \in[1, \infty), N \geq\left[n\left(q_{\omega} / p-1\right)\right]+$ 1 , and $p \in(0,1]$, then

$$
\left\|\mathscr{M}_{N}^{0} f\right\|_{L_{\omega}^{p, \infty}\left(\mathbb{R}^{n}\right)} \sim\left\|\mathscr{M}_{N} f\right\|_{L_{\omega}^{p, \infty}\left(\mathbb{R}^{n}\right)} \sim\left\|\widetilde{\mathscr{M}}_{N} f\right\|_{L_{\omega}^{p, \infty}\left(\mathbb{R}^{n}\right)} \cdot
$$

Proof. The proof of (i) is trivial. For (ii), since $\mathscr{M}_{N}^{0}(f)(x) \leq$ $\mathscr{M}_{N}(f)(x) \leq \widetilde{\mathscr{M}}_{N}(f)(x)$, it is easy to see that

$$
\left\|\mathscr{M}_{N}^{0} f\right\|_{L_{\omega}^{p, \infty}\left(\mathbb{R}^{n}\right)} \leq\left\|\mathscr{M}_{N} f\right\|_{L_{\omega}^{p, \infty}\left(\mathbb{R}^{n}\right)} \leq\left\|\widetilde{M}_{N} f\right\|_{L_{\omega}^{p, \infty}\left(\mathbb{R}^{n}\right)} .
$$

Hence, it suffices to prove that there exists a positive constant $C$ such that

$$
\left\|\widetilde{\mathscr{M}}_{N} f\right\|_{L_{\omega}^{p, \infty}\left(\mathbb{R}^{n}\right)} \leq C\left\|\mathscr{M}_{N}^{0} f\right\|_{L_{\omega}^{p, \infty}\left(\mathbb{R}^{n}\right)} .
$$

By (3.19) of [13], for any $\psi \in \mathscr{D}_{N}^{0}\left(\mathbb{R}^{n}\right), 0<r<1$ satisfying $A>n / r$ and $x \in \mathbb{R}^{n}$, we have

$$
\begin{aligned}
{\left[\psi_{0, A, B}^{* *}(f)(x)\right]^{r} \lesssim } & M^{\mathrm{loc}}\left(\left[\psi_{0}^{+}(f)\right]^{r}\right)(x) \\
& +K_{B r}\left(\left[\psi_{0}^{+}(f)\right]^{r}\right)(x) .
\end{aligned}
$$

By (3.28) of [13], we know that

$$
\widetilde{\mathscr{M}}_{N}(f)(x) \lesssim \psi_{0, A, B}^{* *}(f)(x) .
$$

Therefore, by (26), (27), and $\psi_{0}^{+}(f)(x) \leq \mathscr{M}_{N}^{0} f(x)$, to get (25), it suffices to prove that for $0<r<p \leq 1$ and $q<p / r$ there exists a constant $B$ depending only on $n, r, p, \omega$ such that

$$
\begin{aligned}
& \left\|\left(M^{\mathrm{loc}}\left(g^{r}\right)\right)^{1 / r}\right\|_{L_{\omega}^{p, \infty}\left(\mathbb{R}^{n}\right)} \leqslant\|g\|_{L_{\omega}^{p, \infty}\left(\mathbb{R}^{n}\right)}, \\
& \left\|\left(K_{B r}\left(g^{r}\right)\right)^{1 / r}\right\|_{L_{\omega}^{p, \infty}\left(\mathbb{R}^{n}\right)} \lesssim\|g\|_{L_{\omega}^{p, \infty}\left(\mathbb{R}^{n}\right)} .
\end{aligned}
$$

We first prove (28). For any $t>0$, we set $g=g_{1}+g_{2}$, and $g_{1}(x)=g(x)$ if $|g(x)| \leq t / 2$, otherwise is zero. Without loss of generality, we can assume that $q>1$. By the boundedness of $M^{\text {loc }}$ (see (vii) of Lemma 3 ) and the fact that $r q<p$, we get

$$
\begin{aligned}
& \omega\left(\left\{x \in \mathbb{R}^{n}:\left(M^{\text {loc }}\left(g^{r}\right)(x)\right)^{1 / r}>t\right\}\right) \\
& \leq \omega\left(\left\{x \in \mathbb{R}^{n}: M^{\text {loc }}\left(g_{2}^{r}\right)(x)>\left(\frac{t}{2}\right)^{r}\right\}\right) \\
& \leq t^{-r q} \int_{\left\{x \in \mathbb{R}^{n}:|g(x)| \geq t / 2\right\}}|g(x)|^{r q} \omega(x) d x \\
& \leq t^{-r q} \int_{t / 2}^{\infty} \omega\left(\left\{x \in \mathbb{R}^{n}:|g(x)|>\lambda\right\}\right) d \lambda^{r q} \\
& \quad+t^{-r q} \int_{0}^{t / 2} \omega\left(\left\{x \in \mathbb{R}^{n}:|g(x)|>\frac{t}{2}\right\}\right) d \lambda^{r q} \\
& \leq t^{-r q} \int_{t / 2}^{\infty} \lambda^{r q-1-p} \lambda^{p} \omega\left(\left\{x \in \mathbb{R}^{n}:|g(x)|>\lambda\right\}\right) d \lambda^{r q} \\
& \quad+t^{-p}\|g\|_{L_{\omega}^{p, \infty}\left(\mathbb{R}^{n}\right)}^{p} \leqslant t^{-p}\|g\|_{L_{\omega}^{p, \infty}\left(\mathbb{R}^{n}\right)}^{p}
\end{aligned}
$$

Next we prove (29). By Lemma 7, we have

$$
\left\|K_{B r} g\right\|_{L_{\omega}^{q}\left(\mathbb{R}^{n}\right)} \lesssim\|g\|_{L_{\omega}^{q}\left(\mathbb{R}^{n}\right)},
$$

when $\mathrm{Br}$ is taken to be sufficiently large. Then by (31) and the same method of proof of (28), we obtain (29). The proof of lemma is complete.

\section{The Decomposition Theorem}

Let $0<p \leq 1,1 \leq q<\infty, \omega \in A_{q}^{\rho, \infty}\left(\mathbb{R}^{n}\right)$, and $N \geq\left[n\left(q_{\omega} / p-\right.\right.$ $1)]+1$ with $q_{\omega}$ as in (12). The weighted local Hardy spaces $h_{\rho}^{p}(\omega)$ can be defined by

$$
h_{\rho}^{p}(\omega) \equiv\left\{f \in \mathscr{D}^{\prime}\left(\mathbb{R}^{n}\right): \mathscr{M}_{N}(f) \in L_{\omega}^{p}\left(\mathbb{R}^{n}\right)\right\}
$$

and $\|f\|_{h_{\rho}^{p}(\omega)}=\left\|\mathscr{M}_{N}(f)\right\|_{L_{\omega}^{p}\left(\mathbb{R}^{n}\right)}$. For $h_{\rho}^{p}(\omega)$, we have following lemma.

Lemma 9 (see [13]). Let $\omega \in A_{\infty}^{\rho, \infty}\left(\mathbb{R}^{n}\right)$, then what follows are equivalent:

(i) $f \in h_{\rho}^{p}(\omega)$;

(ii) $f \in \mathscr{D}^{\prime}\left(\mathbb{R}^{n}\right)$ and $\widetilde{\mathscr{M}}_{N}(f) \in L_{\omega}^{p}\left(\mathbb{R}^{n}\right)$;

(iii) $f \in \mathscr{D}^{\prime}\left(\mathbb{R}^{n}\right)$ and $\widetilde{\mathscr{M}}_{N}^{0}(f) \in L_{\omega}^{p}\left(\mathbb{R}^{n}\right)$;

(iv) $f \in \mathscr{D}^{\prime}\left(\mathbb{R}^{n}\right)$ and $\mathscr{M}_{N}^{0}(f) \in L_{\omega}^{p}\left(\mathbb{R}^{n}\right)$.

Moreover, for all $f \in h_{\rho}^{p}(\omega)$,

$$
\begin{aligned}
\|f\|_{h_{\rho}^{p}(\omega)} & \sim\left\|\widetilde{M}_{N}(f)\right\|_{L_{\omega}^{p}\left(\mathbb{R}^{n}\right)} \sim\left\|\widetilde{M}_{N}^{0}(f)\right\|_{L_{\omega}^{p}\left(\mathbb{R}^{n}\right)} \\
& \sim\left\|\mathscr{M}_{N}^{0}(f)\right\|_{L_{\omega}^{p}\left(\mathbb{R}^{n}\right)},
\end{aligned}
$$

where the implicit constants are independent of $f$. 
Similarly, the weighted weak local Hardy spaces $\operatorname{Wh}_{\rho}^{p}(\omega)$ can be defined by

$$
\mathrm{Wh}_{\rho}^{p}(\omega) \equiv\left\{f \in \mathscr{D}^{\prime}\left(\mathbb{R}^{n}\right): \mathscr{M}_{N}(f) \in L_{\omega}^{p, \infty}\left(\mathbb{R}^{n}\right)\right\},
$$

and $\|f\|_{\mathrm{Wh}_{\rho}^{p}(\omega)}=\left\|\mathscr{M}_{N}(f)\right\|_{L_{\omega}^{p, \infty}\left(\mathbb{R}^{n}\right)}$.

In this section, we establish a decomposition theorem of weighted weak local Hardy spaces $\mathrm{Wh}_{\rho}^{p}(\omega)$.

We first recall the Calderón-Zygmund decomposition of $f$ of degree $s$ and height $\lambda$ associated with $\mathscr{M}_{N}(f)$ as in [12, $13,25]$.

Let $\omega \in A_{\infty}^{\rho, \infty}\left(\mathbb{R}^{n}\right)$ and $q_{\omega}$ be as in (12). Throughout this section, we consider a distribution $f$ so that for all $\lambda>0$

$$
\omega\left(\left\{x \in \mathbb{R}^{n}: \mathscr{M}_{N}(f)(x)>\lambda\right\}\right)<\infty .
$$

For a given $\lambda>\inf _{x \in \mathbb{R}^{n}} \mathscr{M}_{N}(f)(x)$, we set

$$
\Omega_{\lambda} \equiv\left\{x \in \mathbb{R}^{n}: \mathscr{M}_{N}(f)(x)>\lambda\right\} .
$$

It is obvious that $\Omega_{\lambda}$ is a proper open subset of $\mathbb{R}^{n}$. As in [4], we give the usual Whitney decomposition of $\Omega_{\lambda}$. Thus we can find closed cubes $Q_{k}$ with $\Omega_{\lambda}=\bigcup_{k} Q_{k}$, and their interiors are away from $\Omega_{\lambda}^{\complement}$ and

$$
\operatorname{diam}\left(Q_{k}\right) \leq 2^{-(6+n)} \operatorname{dist}\left(Q_{k}, \Omega_{\lambda}^{\complement}\right) \leq 4 \operatorname{diam}\left(Q_{k}\right) .
$$

In what follows, fix $a \equiv 1+2^{-(11+n)}$ and $b \equiv 1+2^{-(10+n)}$, and if we denote $\bar{Q}_{k}=a Q_{k}, Q_{k}^{*}=b Q_{k}$, we have $Q_{k} \subset \bar{Q}_{k} \subset Q_{k}^{*}$. Moreover, $\Omega_{\lambda}=\bigcup_{k} Q_{k}^{*}$, and $\left\{Q_{k}^{*}\right\}_{k}$ have the bounded interior property; namely, every point in $\Omega_{\lambda}$ is contained in at most a fixed number of $\left\{Q_{k}^{*}\right\}_{k}$.

Now we take a function $\xi \in \mathscr{D}\left(\mathbb{R}^{n}\right)$ such that $0 \leq \xi \leq 1$, $\operatorname{supp}(\xi) \subset a Q(0,1)$, and $\xi \equiv 1$ on $Q(0,1)$. For $x \in \mathbb{R}^{n}$, set $\xi_{k}(x) \equiv \xi\left(\left(x-x_{k}\right) / l_{k}\right)$, where, and in what follows, $x_{k}$ is the center of the cube $Q_{k}$ and $l_{k}$ is its side length. Obviously, by the construction of $\left\{Q_{k}^{*}\right\}_{k}$ and $\left\{\xi_{k}\right\}_{k}$, for any $x \in \mathbb{R}^{n}$, we have $1 \leq \sum_{k} \xi_{k}(x) \leq M$, where $M$ is a fixed positive integer independent of $x$. Let $\eta_{k} \equiv \xi_{k} /\left(\sum_{j} \xi_{j}\right)$. Then $\left\{\eta_{k}\right\}_{k}$ form a smooth partition of unity for $\Omega_{\lambda}$ subordinate to the locally finite covering $\left\{Q_{k}^{*}\right\}_{k}$ of $\Omega_{\lambda}$; namely, $\chi_{\Omega_{\lambda}}=\sum_{k} \eta_{k}$ with each $\eta_{k} \in \mathscr{D}\left(\mathbb{R}^{n}\right)$ supported in $\bar{Q}_{k}$.

Let $s \in \mathbb{Z}_{+}$be some fixed integer and $\mathscr{P}_{s}\left(\mathbb{R}^{n}\right)$ denote the linear space of polynomials in $n$ variables of degrees no more than $s$. For each $i \in \mathbb{N}$ and $P \in \mathscr{P}_{s}\left(\mathbb{R}^{n}\right)$, set

$$
\|P\|_{i} \equiv\left[\frac{1}{\int_{\mathbb{R}^{n}} \eta_{i}(y) d y} \int_{\mathbb{R}^{n}}|P(x)|^{2} \eta_{i}(x) d x\right]^{1 / 2} .
$$

Then it is easy to see that $\left(\mathscr{P}_{s}\left(\mathbb{R}^{n}\right),\|\cdot\|_{i}\right)$ is a finite dimensional Hilbert space. Let $f \in \mathscr{D}^{\prime}\left(\mathbb{R}^{n}\right)$, since $f$ induces a linear functional on $\mathscr{P}_{s}\left(\mathbb{R}^{n}\right)$ via

$$
P \longmapsto \frac{1}{\int_{\mathbb{R}^{n}} \eta_{i}(y) d y}\left\langle f, P \eta_{i}\right\rangle
$$

by the Riesz representation theorem; there exists a unique polynomial $P_{i} \in \mathscr{P}_{s}\left(\mathbb{R}^{n}\right)$ for each $i$ such that, for all $Q \in$ $\mathscr{P}_{s}\left(\mathbb{R}^{n}\right)$,

$$
\left\langle f, Q \eta_{i}\right\rangle=\left\langle P_{i}, Q \eta_{i}\right\rangle=\int_{\mathbb{R}^{n}} P_{i}(x) Q(x) \eta_{i}(x) d x .
$$

For each $i$, define the distribution $b_{i} \equiv\left(f-P_{i}\right) \eta_{i}$ when $l_{i} \in$ $\left(0, L_{3} \rho\left(x_{i}\right)\right.$ ) (where $L_{3}=2^{k_{0}} C_{0}$ and $x_{i}$ is the center of the cube $\left.Q_{i}\right)$ and $b_{i} \equiv f \eta_{i}$ when $l_{i} \in\left[L_{3} \rho\left(x_{i}\right), \infty\right)$.

As in [13], we can show that for suitable choices of $s$ and $N$, the series $\sum_{i} b_{i}$ converge in $\mathscr{D}^{\prime}\left(\mathbb{R}^{n}\right)$, and, in this case, we define $g \equiv f-\sum_{i} b_{i}$ in $\mathscr{D}^{\prime}\left(\mathbb{R}^{n}\right)$. We point out that the representation $f=g+\sum_{i} b_{i}$, where $g$ and $b_{i}$ are as above, is called a Calderón-Zygmund decomposition of $f$ of degree $s$ and height $\lambda$ associated with $\mathscr{M}_{N}(f)$.

To obtain the main theorem, we need the following lemmas (Lemmas 10-13) about Calderón-Zygmund decomposition which have been given in Section 4 of [13].

Lemma 10. There exists a constant $C>0$ such that

$$
\mathscr{M}_{N}^{0} b_{i}(x) \leq C \mathscr{M}_{N} f(x) \quad \text { for } x \in Q_{i}^{*} .
$$

Lemma 11. Suppose $0 \leq s<N$. Then there exist positive constants $C_{1}$ so that for $i \in \mathbb{N}$

$$
\begin{array}{r}
\mathscr{M}_{N}^{0}\left(b_{i}\right)(x) \leq C \frac{\lambda l_{i}^{n+s+1}}{\left(l_{i}+\left|x-x_{i}\right|\right)^{n+s+1}} \chi_{\left\{\left|x-x_{i}\right|<C_{1} \rho(x)\right\}}(x) \\
\text { if } x \notin Q_{i}^{*} .
\end{array}
$$

Lemma 12. Let $\omega \in A_{\infty}^{\rho, \infty}\left(\mathbb{R}^{n}\right)$ and $q_{\omega}$ be as in (12). If $p \in$ $(0,1], s \geq\left[n\left(q_{\omega} / p-1\right)\right]$, and $N>s$ then there exists a positive constant $C$ such that, for all $f \in h_{\rho}^{p}(\omega), \lambda>\inf _{x \in \mathbb{R}^{n}} \mathscr{M}_{N} f(x)$, and $i \in \mathbb{N}$,

$$
\begin{aligned}
& \int_{\mathbb{R}^{n}}\left(\mathscr{M}_{N}^{0}\left(b_{i}\right)(x)\right)^{p} \omega(x) d x \\
& \quad \leq C \int_{Q_{i}^{*}}\left(\mathscr{M}_{N}(f)(x)\right)^{p} \omega(x) d x .
\end{aligned}
$$

Moreover the series $\sum_{i} b_{i}$ converges in $h_{\rho}^{p}(\omega)$ and

$$
\begin{aligned}
& \int_{\mathbb{R}^{n}}\left(\mathscr{M}_{N}^{0}\left(\sum_{i} b_{i}\right)(x)\right)^{p} \omega(x) d x \\
& \quad \leq C \int_{\Omega}\left(\mathscr{M}_{N}(f)(x)\right)^{p} \omega(x) d x .
\end{aligned}
$$

Lemma 13. Let $\omega \in A_{\infty}^{\rho, \infty}\left(\mathbb{R}^{n}\right)$ and $q_{\omega}$ be as in (12), $q \in$ $\left(q_{\omega}, \infty\right)$, and $p \in(0,1]$.

(i) If $N>s \geq\left[n\left(q_{\omega} / p-1\right)\right]$ and $f \in h_{\rho}^{p}(\omega)$, then $\mathscr{M}_{N}^{0}(g) \in L_{\omega}^{q}\left(\mathbb{R}^{n}\right)$ and there exists a positive constant $C$, independent of $f$ and $\lambda$, such that

$$
\begin{aligned}
& \int_{\mathbb{R}^{n}}\left[\mathscr{M}_{N}^{0}(g)(x)\right]^{q} \omega(x) d x \\
& \quad \leq C \lambda^{q-p} \int_{\mathbb{R}^{n}}\left[\mathscr{M}_{N}(f)(x)\right]^{p} \omega(x) d x .
\end{aligned}
$$

(ii) If $N \geq 2$ and $f \in L_{\omega}^{q}\left(\mathbb{R}^{n}\right)$, then $g \in L_{\omega}^{\infty}\left(\mathbb{R}^{n}\right)$ and there exists a positive constant $C$, independent of $f$ and $\lambda$, such that $\|g\|_{L_{\omega}^{\infty}} \leq C \lambda$. 
Theorem 14. Let $0<p \leq 1,1 \leq q<\infty$, and $\omega \in$ $A_{q}^{\rho, \infty}\left(\mathbb{R}^{n}\right)$, then, for any $f \in W h_{\rho}^{p}(\omega)$, there exists a sequence of bounded function $\left\{f_{m}\right\}_{m=m^{\prime}}^{+\infty}$ with the following properties, in which $m^{\prime} \in \mathbb{Z}$ such that $2^{m^{\prime}-1} \leq \inf _{x \in \mathbb{R}^{n}} \mathscr{M}_{N} f(x)<2^{m^{\prime}}$, and if $\inf _{x \in \mathbb{R}^{n}} \mathscr{M}_{N} f(x)=0$, one writes $m^{\prime}=-\infty$.

(i) $f=\sum_{m=m^{\prime}}^{+\infty} f_{m}$ is in the sense of distribution.

(ii) Each $f_{m}$ can be further decomposed as $f_{m}=\sum_{i} h_{i}^{m}$, where $\left\{h_{i}^{m}\right\}_{i}$ satisfy the fact that

(1) each $h_{i}^{m}$ is supported in a cube $Q_{i}^{m}=Q\left(x_{i}^{m}, r_{i}^{m}\right)$ with $r_{i}^{m} \leq L_{1} \rho\left(x_{i}^{m}\right), \sum_{i} \omega\left(Q_{i}^{m}\right) \leq \widetilde{A} 2^{-m p}$, and $\sum_{i} \chi_{Q_{i}^{m}} \leqslant 1$, where $L_{1} \equiv 4 C_{0}(3 \sqrt{n})^{k_{0}}, \widetilde{A}$ is a constant depending on $f$, and $\chi_{\mathrm{Q}_{i}^{m}}$ denote the characteristic functions;

(2) $\left\|h_{i}^{m}\right\|_{L^{\infty}} \lesssim 2^{m}$ and $\int_{\mathbb{R}^{n}} h_{i}^{m}(x) x^{\alpha} d x=0$ for $\alpha \epsilon$ $\left(\mathbb{Z}_{+}\right)^{n}$ with $|\alpha| \geq\left[n\left(q_{\omega} / p-1\right)\right]+1$, when $r_{i}^{m}<$ $L_{2} \rho\left(x_{i}^{m}\right)$ and $L_{2} \equiv 1 / C_{0}^{2}(3 \sqrt{n})^{k_{0}+1}$.

Conversely, if a distribution $f$ satisfies (i) and (ii), then $f \in$ $W h_{\rho}^{p}(\omega)$.

Moreover, one has $\|f\|_{W h_{\rho}^{p}(\omega)}^{p} \sim \widetilde{A}$.

Proof. We first suppose $f \in \mathrm{Wh}_{\rho}^{p}(\omega)$, and set $\Omega_{m} \equiv\left\{x \in \mathbb{R}^{n}\right.$ : $\left.\mathscr{M}_{N} f(x)>2^{m}\right\}$. As above, let $\Omega_{m}=\bigcup_{i} \widetilde{Q}_{i}^{m}=\bigcup_{i} Q\left(\widetilde{x}_{i}^{m}, \widetilde{r}_{i}^{m}\right)$ be the Whitney decomposition, and we write $\left\{Q_{i}\right\}_{i},\left\{\eta_{i}\right\}_{i},\left\{P_{i}\right\}_{i}$, and $\left\{b_{i}\right\}_{i}$, respectively, as $\left\{\widetilde{Q}_{i}^{m}\right\}_{i},\left\{\eta_{i}^{m}\right\}_{i},\left\{P_{i}^{m}\right\}_{i}$, and $\left\{b_{i}^{m}\right\}_{i}$; that is, $b_{i}^{m} \equiv\left(f-P_{i}^{m}\right) \eta_{i}^{m}$ if $\widetilde{r}_{i}^{m}<L_{3} \rho\left(\widetilde{x}_{i}^{m}\right), b_{i}^{m} \equiv f \eta_{i}^{m}$ if $\widetilde{r}_{i}^{m} \geq L_{3} \rho\left(\widetilde{x}_{i}^{m}\right)$, and $\eta_{i}^{m}$ is a smooth function supported in $\widetilde{Q}_{i}^{m *} \equiv b \widetilde{Q}_{i}^{m}$. Then by Lemmas 10 and 11, there exists a constant $C_{1}$ such that for any $i \in \mathbb{N}$

$$
\begin{aligned}
\mathscr{M}_{N}^{0}\left(b_{i}^{m}\right)(x) \\
\leq \mathscr{M}_{N} f(x) \chi_{\widetilde{Q}_{i}^{m *}}(x) \\
\quad+\frac{2^{m}\left(\widetilde{r}_{i}^{m}\right)^{n+N}}{\left(\widetilde{r}_{i}^{m}+\left|x-\widetilde{x}_{i}^{m}\right|\right)^{n+N}} \chi_{\left\{\left|x-\widetilde{x}_{i}^{m}\right|<C_{1} \rho\left(\widetilde{x}_{i}^{m}\right)\right\} \cap\left(\widetilde{Q}_{i}^{m *}\right)^{c}}(x),
\end{aligned}
$$

where $N \geq\left[n\left(q_{\omega} / p-1\right)\right]+2$.

Then by Lemma 9 and using the similar method of proof of Lemma 12, we have

$$
\begin{aligned}
& \left\|\sum_{i} b_{i}^{m}\right\|_{h_{\rho}^{p_{0}}(\omega)}^{p_{0}} \lesssim \int_{\mathbb{R}^{n}}\left(\mathscr{M}_{N}^{0}\left(\sum_{i} b_{i}\right)(x)\right)^{p_{0}} \omega(x) d x \\
& \leq \int_{\Omega_{m}}\left(\mathscr{M}_{N}(f)(x)\right)^{p_{0}} \omega(x) d x \\
& \leq \int_{2^{m}}^{\infty} \omega\left(\left\{x \in \mathbb{R}^{n}: \mathscr{M}_{N}(f)(x)>\lambda\right\}\right) d \lambda^{p_{0}} \\
& \quad+\int_{0}^{2^{m}} \omega\left(\left\{x \in \mathbb{R}^{n}: \mathscr{M}_{N}(f)(x)>2^{m}\right\}\right) d \lambda^{p_{0}} \\
& \leq\|f\|_{\mathrm{Wh}_{\rho}^{p}(\omega)}^{p} 2^{m\left(p_{0}-p\right)}, \quad \frac{n}{n+N}<p_{0}<p .
\end{aligned}
$$

Hence, $\sum_{i} b_{i}^{m}$ converges in the sense of distributions, and we have the Calderón-Zygmund decomposition $f=g^{m}+$ $\sum_{i} b_{i}^{m}$. By using the similar method of proof of Lemma 13(ii), we have $\left\|g^{m}\right\|_{L_{\omega}^{\infty}} \leqslant 2^{m}$. Let $f_{m}=g^{m+1}-g^{m}$; then $\sum_{i} \eta_{i}^{m} b_{j}^{m+1}=$ $\chi_{\Omega_{m}} b_{j}^{m+1}=b_{j}^{m+1}$ for all $j$ and

$$
\begin{aligned}
g^{m+1}-g^{m} & =\left(f-\sum_{j} b_{j}^{m+1}\right)-\left(f-\sum_{i} b_{i}^{m}\right) \\
& =\sum_{i} b_{i}^{m}-\sum_{j} b_{j}^{m+1} \\
& =\sum_{i}\left[b_{i}^{m}-\sum_{j \in F_{1}^{m}} b_{j}^{m+1} \eta_{i}^{m}+\sum_{j \in F_{2}^{m}} b_{j}^{m+1} \eta_{i}^{m}\right] \\
& \equiv \sum_{i} h_{i}^{m},
\end{aligned}
$$

where $F_{1}^{m} \equiv\left\{i \in \mathbb{N}: \widetilde{r}_{i}^{m} \geq L_{3} \rho\left(\widetilde{x}_{i}^{m}\right)\right\}, F_{2}^{m} \equiv\left\{i \in \mathbb{N}: \widetilde{r}_{i}^{m}<\right.$ $\left.L_{3} \rho\left(\widetilde{x}_{i}^{m}\right)\right\}$, and all the series converges in $\mathscr{D}^{\prime}\left(\mathbb{R}^{n}\right)$.

We set $\operatorname{supph}_{i}^{m} \subset Q_{i}^{m} \equiv Q\left(x_{i}^{m}, r_{i}^{m}\right)$. By the similar method of Lemma 5.4 in [13], if $r_{i}^{m}<L_{2} \rho\left(x_{i}^{m}\right)$, it is easy to see that $h_{i}^{m}$ satisfy all conditions in (ii); if $r_{i}^{m} \geq L_{2} \rho\left(x_{i}^{m}\right)$, we can decompose $Q_{i}^{m}$ into a finite number of disjoint cubes $\left\{Q_{i, m}^{j}\right\}_{j=1}^{N_{i m}}$, and the side length of each cube is between $L_{2} \rho\left(x_{i}^{m}\right)$ and $L_{1} \rho\left(x_{i}^{m}\right)$, and then $\left\{h_{i}^{m} \chi_{Q_{i, m}^{j}}\right\}_{j=1}^{N_{i m}}$ satisfy all conditions in (b). Obviously, $f=\sum_{m=m^{\prime}}^{+\infty} f_{m}$ is also in the sense of distribution.

For the converse, take $\lambda>0$ and $m_{0} \in \mathbb{Z}$ such that $2^{m_{0}} \leq$ $\lambda<2^{k_{0}}$. Without loss of generality, we assume that $m^{\prime}=-\infty$ and write

$$
f=\sum_{m=m^{\prime}}^{m_{0}} f_{m}+\sum_{m=m_{0}+1}^{+\infty} f_{m} \equiv F_{1}+F_{2} .
$$

Then we have $\mathscr{M}_{N}^{0} F_{1}(x) \lesssim \lambda$, and we only need to prove

$$
\omega\left(\left\{x \in \mathbb{R}^{n}: \mathscr{M}_{N}^{0} F_{2}(x)>\lambda\right\}\right) \leqslant \widetilde{A} \lambda^{-p} .
$$

Taking $a_{1}=\sqrt{n} L_{1}+C_{0}^{2} 2^{k_{0}}\left(1+L_{1}\right)$, where $C_{0}$ and $k_{0}$ are constants given in Lemma 1 , then let $\bar{Q}_{i}^{m}=Q\left(x_{i}^{m}, l_{i}^{m}\right)$, where $l_{i}^{m}=\min \left\{2(3 / 2)^{\left(m-m_{0}\right) p / q n} r_{i}^{m}, a_{1} \rho\left(x_{i}^{m}\right)\right\}$ and set

$$
\Omega^{m_{0}} \equiv \bigcup_{m=m_{0}+1}^{+\infty} \bigcup_{i} \bar{Q}_{i}^{m}
$$

By the properties of $A_{q}^{\rho, \infty}\left(\mathbb{R}^{n}\right)$, we have

$$
\omega\left(\bar{Q}_{i}^{m}\right) \lesssim\left(\frac{3}{2}\right)^{\left(m-m_{0}\right) p} \omega\left(Q_{i}^{m}\right)
$$


then by (1) of (ii), we get

$$
\begin{aligned}
\omega\left(\Omega^{m_{0}}\right) & \lesssim \sum_{m=m_{0}+1}^{+\infty} \sum_{i}\left(\frac{3}{2}\right)^{\left(m-m_{0}\right) p} \omega\left(Q_{i}^{m}\right) \\
& \lesssim \sum_{m=m_{0}+1}^{+\infty} 2^{-\left(m-m_{0}\right) p}\left(\frac{3}{2}\right)^{\left(m-m_{0}\right) p} 2^{-m_{0} p} \widetilde{A} \\
& \lesssim \lambda^{-p} \widetilde{A} .
\end{aligned}
$$

Hence, to prove (50), it suffices to prove

$$
\int_{\left(\Omega^{m_{0}}\right)^{\complement}}\left(\mathscr{M}_{N}^{0} F_{2}(x)\right)^{p} \omega(x) d x \leqslant \widetilde{A} .
$$

Then, we just need to estimate

$$
\int_{\left(\bar{Q}_{i}^{m}\right)^{c}}\left(\mathscr{M}_{N}^{0} h_{i}^{m}(x)\right)^{p} \omega(x) d x .
$$

In fact, let $x \in\left(\bar{Q}_{i}^{m}\right)^{\complement}$; we have the following estimate:

$$
\begin{aligned}
& \mathscr{M}_{N}^{0} h_{i}^{m}(x) \\
& \quad \lesssim 2^{m}\left(r_{i}^{m}\right)^{n+N}\left|x-x_{i}^{m}\right|^{-(n+N)} \chi_{\left\{\left|x-x_{i}^{m}\right|<a_{1} \rho\left(x_{i}^{m}\right)\right\}}(x),
\end{aligned}
$$

where $N \geq\left[n\left(q_{\omega} / p-1\right)\right]+1$. From this, and note that $\omega \in$ $A_{q_{\omega}+\varepsilon}^{\rho, \infty}$, for any $\varepsilon>0$, we obtain

$$
\begin{aligned}
& \int_{\left(\overline{\mathrm{Q}}_{i}^{m}\right)^{c}}\left(\mathscr{M}_{N}^{0} h_{i}^{m}(x)\right)^{p} \omega(x) d x \\
& \quad \lesssim \int_{(3 / 2)^{\left(m-m_{0}\right) p / q n} r_{i}^{m}<\left|x-x_{i}^{m}\right|<a_{1} \rho\left(x_{i}^{m}\right)} \frac{2^{m p}\left(r_{i}^{m}\right)^{p(n+N)}}{\left|x-x_{i}^{m}\right|^{p(n+N)}} \omega(x) d x \\
& \quad \lesssim 2^{m p}\left(\frac{2}{3}\right)^{l_{N}\left(m-m_{0}\right) p / q n} \omega\left(Q_{i}^{m}\right),
\end{aligned}
$$

when $\varepsilon$ is small enough such that $l_{N} \equiv p(n+N)-n\left(q_{\omega}+\varepsilon\right)>0$.

Therefore, we get

$$
\begin{aligned}
& \int_{\left(\Omega^{m}\right)^{\complement}}\left(\mathscr{M}_{N}^{0} F_{2}(x)\right)^{p} \omega(x) d x \\
& \lesssim \sum_{m=m_{0}+1}^{\infty} 2^{m p}\left(\sum_{i}\left(\frac{2}{3}\right)^{l_{N}\left(m-m_{0}\right) p / q n} \omega\left(Q_{i}^{m}\right)\right) \\
& \lesssim \sum_{m=m_{0}+1}^{\infty} 2^{m p}\left(\frac{2}{3}\right)^{l_{N}\left(m-m_{0}\right) p / q n}\left(2^{-m p} \widetilde{A}\right) \lesssim \widetilde{A},
\end{aligned}
$$

which infers (54), and the proof is complete.

\section{Application}

In this section, we will show the boundedness of localized Riesz transforms on $\mathrm{Wh}_{\rho}^{p}(\omega)$ spaces. As in $[13,26]$, for all $j \in$ $\{1,2, \ldots, n\}$, and $x \in \mathbb{R}^{n}$, define localized Riesz transforms as

$$
\begin{aligned}
& \widehat{R}_{j}(f)(x) \\
& \quad \equiv \text { p.v. } c_{n} \int_{\mathbb{R}^{n}} \frac{x_{j}-y_{j}}{|x-y|^{n+1}} \eta\left(\frac{|x-y|}{\rho(x)}\right) f(y) d y,
\end{aligned}
$$

where and, in what follows, $c_{n} \equiv \Gamma((n+1) / 2) /\left[\pi^{(n+1) / 2}\right], \eta \in$ $C^{1}\left(\mathbb{R}^{n}\right)$ supported in $(-1,1)$, and $\eta(t)=1$ if $|t| \leq 1 / 2$.

As in [12], we can obtain the following lemma. Its proof is similar to Lemma 8.2 in [12], and we omit the details here.

Lemma 15. Let $\widehat{R}_{j}$ be localized Riesz transforms, where $j \in$ $\{1,2, \ldots, n\}$; then

(i) $\left\|\widehat{R}_{j}(f)\right\|_{L_{\omega}^{p}\left(\mathbb{R}^{n}\right)} \leq C_{p, \omega}\|f\|_{L_{\omega}^{p}\left(\mathbb{R}^{n}\right)}$, for $1<p<\infty$ and $\omega \in A_{p}^{\rho, \infty}\left(\mathbb{R}^{n}\right) ;$

(ii) $\left\|\widehat{R}_{j}(f)\right\|_{L_{\omega}^{1, \infty}\left(\mathbb{R}^{n}\right)} \leq C_{\omega}\|f\|_{L_{\omega}^{1}\left(\mathbb{R}^{n}\right)}$, for $\omega \in A_{1}^{\rho, \infty}\left(\mathbb{R}^{n}\right)$.

Now let us state the main result of this section.

Theorem 16. Let $\omega \in A_{\infty}^{\rho, \infty}\left(\mathbb{R}^{n}\right), 0<p \leq 1$, and $\widehat{R}_{j}$ be localized Riesz transforms, where $j \in\{1,2, \ldots, n\}$; then there is a constant $C_{\omega}$ independent of $f \in W h_{\rho}^{p}(\omega)$ such that

$$
\left\|\widehat{R}_{j} f\right\|_{W h_{\rho}^{p}(\omega)} \leq C_{\omega}\|f\|_{W h_{\rho}^{p}(\omega)} .
$$

Proof. By the definition of $\mathrm{Wh}_{\rho}^{p}(\omega)$, to get (60), it suffices to prove

$$
\begin{gathered}
\omega\left(\left\{x \in \mathbb{R}^{n}: \mathscr{M}_{N}^{0}\left(\widehat{R}_{j} f\right)(x)>\lambda\right\}\right) \\
\leq C_{\omega} \lambda^{-p}\|f\|_{\mathrm{Wh}_{\rho}^{p}(\omega)}^{p},
\end{gathered}
$$

for any $\lambda>0$. Let $f \in \mathrm{Wh}_{\rho}^{p}(\omega)$; then we have the decomposition of $f$ as in Theorem 14. Particularly, we have

$$
f=\sum_{m=m^{\prime}}^{+\infty} f_{m}
$$

without loss of generality; we always assume that $m^{\prime}=-\infty$. Fix $\lambda>0$, and take $m_{0} \in \mathbb{Z}$ such that $2^{m_{0}} \leq \lambda<2^{k_{0}}$; then we write

$$
f=\sum_{m=-\infty}^{m_{0}} f_{m}+\sum_{m=m_{0}+1}^{+\infty} f_{m} \equiv F_{1}+F_{2} .
$$

For $F_{1}$, since $\omega \in A_{q}^{\rho, \infty}$, for some $1<q<\infty$, by Proposition 8 and Theorem 14, we have

$$
\begin{aligned}
\left\|F_{1}\right\|_{L_{\omega}^{q}\left(\mathbb{R}^{n}\right)} & \leq C \sum_{m=-\infty}^{m_{0}} 2^{m} \omega\left(\Omega_{m}\right)^{1 / q} \\
& \leq C\|f\|_{\mathrm{Wh}_{\rho}^{p}(\omega)}^{p / q} \sum_{m=-\infty}^{m_{0}} 2^{m(1-p / q)} \\
& \leq C \lambda^{1-p / q}\|f\|_{\operatorname{Wh}_{\rho}^{p}(\omega)}^{p / q} .
\end{aligned}
$$

Hence, since $\widehat{R}_{j}$ is bounded on $L_{\omega}^{q}\left(\mathbb{R}^{n}\right)$ by (i) of Lemma 15 , we get

$$
\begin{aligned}
& \omega\left(\left\{x \in \mathbb{R}^{n}: \mathscr{M}_{N}^{0}\left(\widehat{R}_{j} F_{1}\right)(x)>\lambda\right\}\right) \leq C \frac{\left\|\widehat{R}_{j} F_{1}\right\|_{L_{\omega}^{q}\left(\mathbb{R}^{n}\right)}^{q}}{\lambda^{q}} \\
& \quad \leq C \frac{\left\|F_{1}\right\|_{L_{\omega}^{q}\left(\mathbb{R}^{n}\right)}^{q}}{\lambda^{q}} \leq C \lambda^{-p}\|f\|_{\operatorname{Wh}_{\rho}^{p}(\omega)}^{p} .
\end{aligned}
$$


Then we just need to prove that

$$
\begin{aligned}
\omega( & \left(\left\{x \in \mathbb{R}^{n}: \mathscr{M}_{N}^{0}\left(\widehat{R}_{j} F_{2}\right)(x)>\lambda\right\}\right) \\
& \leq C_{\omega} \lambda^{-p}\|f\|_{\operatorname{Wh}_{\rho}^{p}(\omega)}^{p} .
\end{aligned}
$$

For each $f_{m}$, by Theorem 14, $f_{m}$ has decomposition $f_{m}=$ $\sum_{i} h_{i}^{m}$, and each $h_{i}^{m}$ is supported in a cube $Q_{i}^{m}=Q\left(x_{i}^{m}, r_{i}^{m}\right)$ with $r_{i}^{m} \leq L_{1} \rho\left(x_{i}^{m}\right)$. Furthermore, there exists a constant $a_{2}>1$ independent of $h_{i}^{m}$ such that $\operatorname{supp} \mathscr{M}_{N}^{0}\left(\widehat{R}_{j} h_{i}^{m}\right)(x) \subset$ $B\left(x_{i}^{m}, a_{2} \rho\left(x_{i}^{m}\right)\right)$. Let $\bar{Q}_{i}^{m}=Q\left(x_{i}^{m}, l_{i}^{m}\right)$, where $l_{i}^{m}=\min \{2(3 /$ 2) $\left.{ }^{\left(m-m_{0}\right) p / q n} r_{i}^{m}, a_{2} \rho\left(x_{i}^{m}\right)\right\}$ and set

$$
\Omega^{m_{0}} \equiv \bigcup_{m=m_{0}+1}^{+\infty} \bigcup_{i} \bar{Q}_{i}^{m} .
$$

By the properties of $A_{q}^{\rho, \infty}\left(\mathbb{R}^{n}\right)$, we have

$$
\omega\left(\bar{Q}_{i}^{m}\right) \lesssim\left(\frac{3}{2}\right)^{\left(m-m_{0}\right) p} \omega\left(Q_{i}^{m}\right) ;
$$

then by Theorem 14, we get

$$
\begin{aligned}
\omega & \left(\Omega^{m_{0}}\right) \lesssim \sum_{m=m_{0}+1}^{+\infty} \sum_{i}\left(\frac{3}{2}\right)^{\left(m-m_{0}\right) p} \omega\left(Q_{i}^{m}\right) \\
& \lesssim \sum_{m=m_{0}+1}^{+\infty} 2^{-\left(m-m_{0}\right) p}\left(\frac{3}{2}\right)^{\left(m-m_{0}\right) p} 2^{-m_{0} p}\|f\|_{\mathrm{Wh}_{\rho}^{p}(\omega)}^{p} \\
& \leqslant \lambda^{-p}\|f\|_{\mathrm{Wh}_{\rho}^{p}(\omega)}^{p} .
\end{aligned}
$$

Therefore, it suffices to prove

$$
\int_{\left(\Omega^{m_{0}}\right)^{\complement}}\left(\mathscr{M}_{N}^{0}\left(\widehat{R}_{j} F_{2}\right)(x)\right)^{p} \omega(x) d x \leq C\|f\|_{\mathrm{Wh}_{\rho}^{p}(\omega)}^{p} .
$$

Then, we just need to estimate

$$
\int_{\left(\bar{Q}_{i}^{m}\right)^{\complement}}\left(\mathscr{M}_{N}^{0}\left(\widehat{R}_{j} h_{i}^{m}\right)(x)\right)^{p} \omega(x) d x .
$$

We will discuss the following two cases.

Case 1. If $L_{2} \rho\left(x_{i}^{m}\right) \leq r_{i}^{m} \leq L_{1} \rho\left(x_{i}^{m}\right)$, let $a_{3} \equiv a_{2} / L_{2}>$ 1 and $Q_{i m}^{*}=a_{3} Q_{i}^{m}$, then since $\operatorname{supp} \mathscr{M}_{N}^{0}\left(\widehat{R}_{j} h_{i}^{m}\right)(x) \subset$ $B\left(x_{i}^{m}, a_{2} \rho\left(x_{i}^{m}\right)\right)$, we have

$$
\begin{aligned}
& \int_{\left(\bar{Q}_{i}^{m}\right)^{c}}\left(\mathscr{M}_{N}^{0}\left(\widehat{R}_{j} h_{i}^{m}\right)(x)\right)^{p} \omega(x) d x \\
& =\left(\int_{Q_{i m}^{*} \backslash \bar{Q}_{i}^{m}}+\int_{\mathbb{R}^{n} \backslash Q_{i m}^{*}}\right)\left(\mathscr{M}_{N}^{0}\left(\widehat{R}_{j} h_{i}^{m}\right)(x)\right)^{p} \omega(x) d x \\
& =\int_{Q_{i m}^{*} \backslash \bar{Q}_{i}^{m}}\left(\mathscr{M}_{N}^{0}\left(\widehat{R}_{j} h_{i}^{m}\right)(x)\right)^{p} \omega(x) d x .
\end{aligned}
$$

Furthermore,

$$
\begin{aligned}
& \int_{Q_{i m}^{*} \backslash \bar{Q}_{i}^{m}}\left(\mathscr{M}_{N}^{0}\left(\widehat{R}_{j} h_{i}^{m}\right)(x)\right)^{p} \omega(x) d x \\
& \leq C 2^{m p} \int_{(3 / 2)^{\left(m-m_{0}\right) p / q n} r_{i}^{m}<\left|x-x_{i}^{m}\right|<a_{3} r_{i}^{m}} \omega(x) d x \\
& \leq C 2^{m p} \omega\left(Q_{i}^{m}\right),
\end{aligned}
$$

for $0 \leq m \leq m_{0}+n q \log _{3 / 2} a_{2}$.

Case 2. If $r_{i}^{m}<L_{2} \rho\left(x_{i}^{m}\right)$, by the vanishing moment of $h_{i}^{m}$, we have

$$
\begin{aligned}
& \mathscr{M}_{N}^{0}\left(\widehat{R}_{j} h_{i}^{m}\right)(x) \\
& \quad \leq C 2^{m} \frac{\left(r_{i}^{m}\right)^{n+N}}{\left|x-x_{i}^{m}\right|^{n+N}} \chi_{\left\{\left|x-x_{i}^{m}\right|<a_{2} \rho\left(x_{i}^{m}\right)\right\}}(x),
\end{aligned}
$$

where $N \geq\left[n\left(q_{\omega} / p-1\right)\right]+1$. From this, and note that $\omega \in$ $A_{q_{\omega}+\varepsilon}^{\rho, \infty}$, for any $\varepsilon>0$, we obtain

$$
\begin{aligned}
& \int_{\left(\bar{Q}_{i}^{m}\right)^{c}}\left(M_{N}^{0}\left(\widehat{R}_{j} h_{i}^{m}\right)(x)\right)^{p} \omega(x) d x \\
& \quad \leqslant \int_{(3 / 2)^{\left(m-m_{0}\right) p / q n} r_{i}^{m}<\left|x-x_{i}^{m}\right|<a_{2} \rho\left(x_{i}^{m}\right)} \frac{2^{m p}\left(r_{i}^{m}\right)^{p(n+N)}}{\left|x-x_{i}^{m}\right|^{p(n+N)}} \omega(x) d x \\
& \quad \leqslant 2^{m p}\left(\frac{2}{3}\right)^{l_{N}\left(m-m_{0}\right) p / q n} \omega\left(Q_{i}^{m}\right),
\end{aligned}
$$

when $\varepsilon$ is small enough such that $l_{N} \equiv p(n+N)-n\left(q_{\omega}+\varepsilon\right)>0$.

Combining the above two cases with Theorem 14, we get

$$
\begin{aligned}
& \int_{\left(\Omega^{m_{0}}\right)^{\complement}}\left(\mathscr{M}_{N}^{0}\left(\widehat{R}_{j} F_{2}\right)(x)\right)^{p} \omega(x) d x \\
& \quad \lesssim \sum_{m=m_{0}+1}^{\infty} 2^{m p}\left(\sum_{i}\left(\frac{2}{3}\right)^{l_{N}\left(m-m_{0}\right) p / q n} \omega\left(Q_{i}^{m}\right)\right) \\
& \quad \lesssim \sum_{m=m_{0}+1}^{\infty} 2^{m p}\left(\frac{2}{3}\right)^{l_{N}\left(m-m_{0}\right) p / q^{n}}\left(2^{-m p}\|f\|_{\mathrm{Wh}_{\rho}^{p}(\omega)}^{p}\right) \\
& \leq\|f\|_{\mathrm{Wh}_{\rho}^{p}(\omega)}^{p} .
\end{aligned}
$$

Therefore, (70) holds, and the proof is complete.

\section{Conflict of Interests}

The author declares that there is no conflict of interests regardng the publication of this paper.

\section{Acknowledgments}

The research is supported by National Natural Science Foundation of China (Tianyuan Foundation of Mathematics) no. 11426038. The author would like to thank the referees for their very valuable suggestions which made this paper more readable. 


\section{References}

[1] D. Goldberg, "A local version of real Hardy spaces," Duke Mathematical Journal, vol. 46, no. 1, pp. 27-42, 1979.

[2] B. Huy Qui, "Weighted Hardy spaces," Mathematische Nachrichten, vol. 103, pp. 45-62, 1981.

[3] T. Schott, "Pseudodifferential operators in function spaces with exponential weights," Mathematische Nachrichten, vol. 200, pp. 119-149, 1999.

[4] E. M. Stein, Harmonic Analysis: Real Variable Methods, Orthogonality, and Oscillatory Integrals, Princeton University Press, Princeton, NJ, USA, 1993.

[5] M. E. Taylor, Pseudodifferential Operators and Nonlinear PDE, vol. 100 of Progress in Mathematics, Birkhäuser, Boston, Mass, USA, 1991.

[6] H. Triebel, Theory of Function Spaces, Birkhäuser, Basel, Switzerland, 1983.

[7] H. Triebel, Theory of Function Spaces II, vol. 84 of Monographs in Mathematics, Birkhäuser, Basel, Switzerland, 1992.

[8] V. S. Rychkov, "Littlewood-Paley theory and function spaces with $A_{p}^{\text {loc }}$ weights," Mathematische Nachrichten, vol. 224, no. 1, pp. 145-180, 2001.

[9] J. Duoandikoetxea, Fourier Analysis, vol. 29 of Graduate Studies in Mathematics, American Mathematical Society, Providence, RI, USA, 2000.

[10] J. García-Cuerva and J. Rubio de Francia, Weighted Norm Inequalities and Related Topics, North-Holland, Amsterdam, The Netherlands, 1985.

[11] L. Grafakos, Classical and Modern Fourier Analysis, Pearson Education, Upper Saddle River, NJ, USA, 2004.

[12] L. Tang, "Weighted local Hardy spaces and their applications," Illinois Journal of Mathematics, vol. 56, no. 2, pp. 453-495, 2012.

[13] H. Zhu and L. Tang, "Weighted local Hardy spaces associated to Schrödinger operators," http://arxiv.org/abs/1403.7641.

[14] R. Fefferman and F. Soria, "The space weak $H^{1}$," Studia Mathematica, vol. 8, pp. 1-16, 1987.

[15] H. Liu, "The weak $H^{p}$ spaces on homogeneous groups," in Harmonic Analysis (Tianjin, 1988), vol. 1494 of Lecture Notes in Mathematics, pp. 113-118, Springer, 1991.

[16] L. Tang, “The weighted weak local Hardy spaces," Rocky Mountain Journal of Mathematics, vol. 44, no. 1, pp. 297-315, 2014.

[17] B. Bongioanni, E. Harboure, and O. Salinas, "Classes of weights related to Schrödinger operators," Journal of Mathematical Analysis and Applications, vol. 373, no. 2, pp. 563-579, 2011.

[18] L. Tang, "Weighted norm inequalities for Schrödinger type operators," Forum Mathematicum, vol. 27, no. 4, pp. 2491-2532, 2015.

[19] L. Tang, "Extrapolation from $A_{\infty}^{\rho, \infty}$, vector-valued inequalities and applications in the Schrödinger settings," Arkiv för Matematik, vol. 25, no. 1, pp. 175-202, 2014.

[20] F. W. Gehring, "The $L^{p}$-integrability of the partial derivatives of a quasiconformal mapping," Acta Mathematica, vol. 130, pp. 265-277, 1973.

[21] J. Dziubański, G. Garrigós, T. Martínez, J. L. Torrea, and J. Zienkiewicz, "BMO spaces related to Schrödinger operators with potentials satisfying a reverse Hölder inequality," Mathematische Zeitschrift, vol. 249, no. 2, pp. 329-356, 2005.

[22] Z. W. Shen, " $L^{p}$ estimates for Schrödinger operators with certain potentials," Annales de l'Institut Fourier, vol. 45, no. 2, pp. 513-546, 1995.
[23] J. Dziubanski and J. Zienkiewicz, "Hardy space $H^{1}$ associated to Schrödinger operator with potential satisfying reverse Hölder inequality," Revista Matemática Iberoamericana, vol. 15, no. 2, pp. 279-296, 1999.

[24] B. Muckenhoupt, "Weighted norm inequalities for the Hardy maximal function," Transactions of the American Mathematical Society, vol. 165, pp. 207-226, 1972.

[25] D. Yang and S. Yang, "Weighted local Orlicz Hardy spaces with applications to pseudo-differential operators," Dissertationes Mathematicae, vol. 478, p. 78, 2011.

[26] D. Yang, D. Yang, and Y. Zhou, "Endpoint properties of localized Riesz transforms and fractional integrals associated to Schrödinger operators," Potential Analysis, vol. 30, no. 3, pp. 271-300, 2009. 


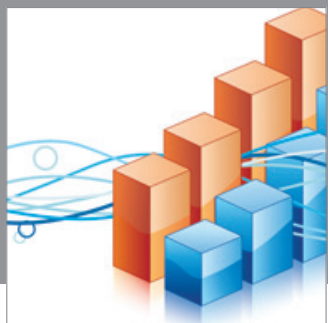

Advances in

Operations Research

mansans

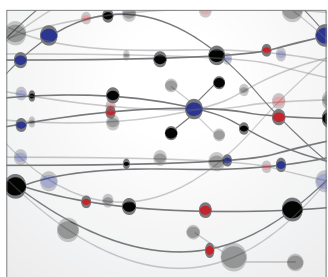

The Scientific World Journal
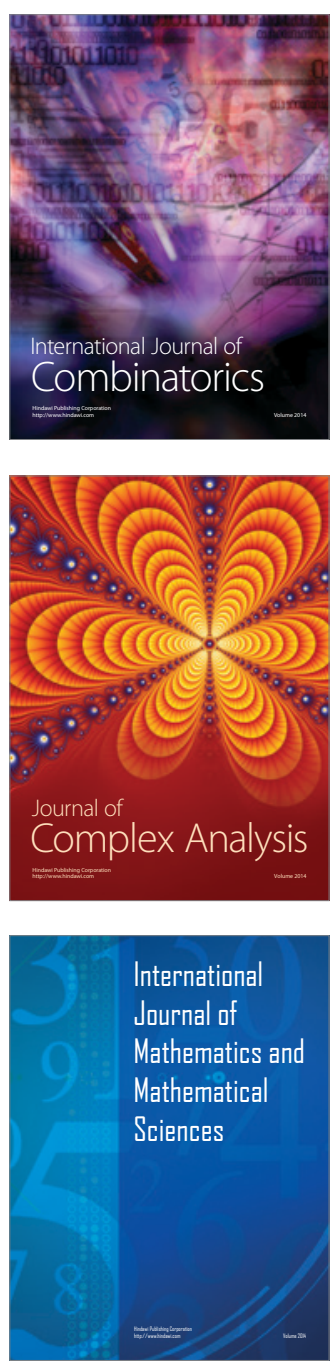
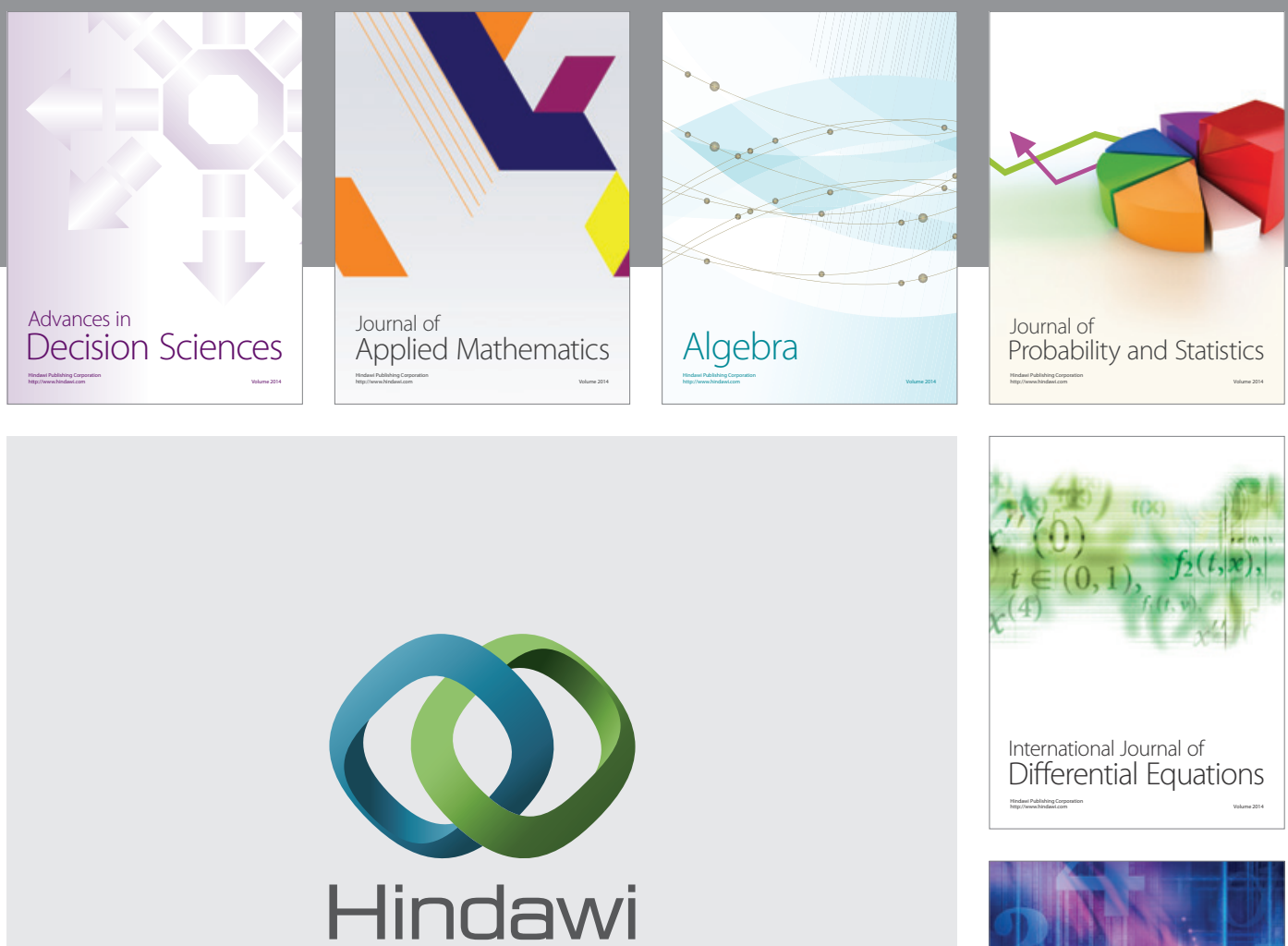

Submit your manuscripts at http://www.hindawi.com
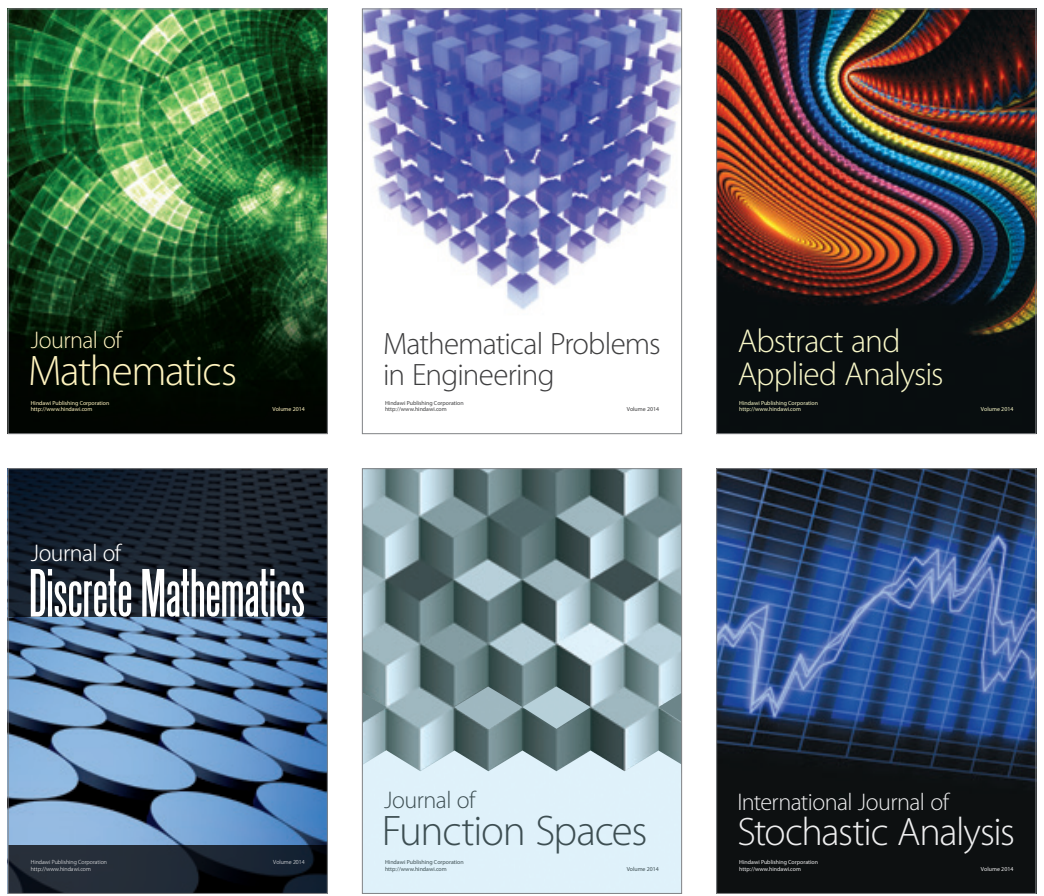

Journal of

Function Spaces

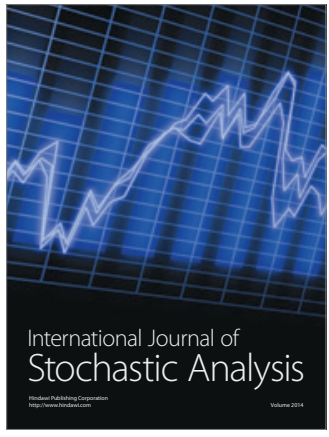

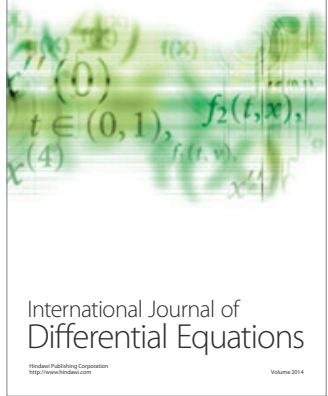
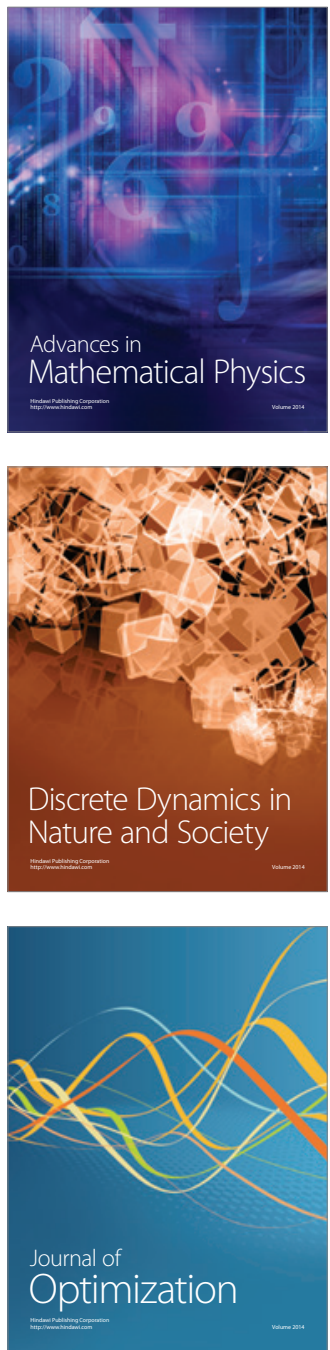